\title{
INTEGRAL MEANS OF THE DERIVATIVES OF BLASCHKE PRODUCTS
}

\author{
EMMANUEL FRICAIN \\ Université de Lyon; Université Lyon 1; Institut Camille Jordan CNRS UMR 5208; \\ 43, boulevard du 11 Novembre 1918, F-69622 Villeurbanne \\ e-mail:fricain@math.univ-lyon1.fr \\ and JAVAD MASHREGHI \\ Département de mathématiques et de statistique, Université Laval, Québec, QC, Canada G1V0A6 \\ e-mail: Javad.Mashreghi@mat.ulaval.ca
}

(Received 10 September, 2007; revised 10 October, 2007; accepted 14 October, 2007)

\begin{abstract}
We study the rate of growth of some integral means of the derivatives of a Blaschke product and we generalize several classical results. Moreover, we obtain the rate of growth of integral means of the derivative of functions in the model subspace $K_{B}$ generated by the Blaschke product $B$.
\end{abstract}

2000 Mathematics Subject Classification. Primary: 30D50, Secondary: 32A70.

1. Introduction. Let $\left(z_{n}\right)_{n \geq 1}$ be a sequence in the unit disc satisfying the Blaschke condition

$$
\sum_{n=1}^{\infty}\left(1-\left|z_{n}\right|\right)<\infty
$$

Then the product

$$
B(z)=\prod_{n=1}^{\infty} \frac{\left|z_{n}\right|}{z_{n}} \frac{z_{n}-z}{1-\bar{z}_{n} z}
$$

is a bounded analytic function on the unit disc $\mathbb{D}$ with zeros only at the points $z_{n}$, $n \geq 1$, [5, p. 20]. Since the product converges uniformly on compact subsets of $\mathbb{D}$, the logarithmic derivative of $B$ is given by

$$
\frac{B^{\prime}(z)}{B(z)}=\sum_{n=1}^{\infty} \frac{1-\left|z_{n}\right|^{2}}{\left(1-\bar{z}_{n} z\right)\left(z-z_{n}\right)}, \quad(z \in \mathbb{D}) .
$$

Therefore,

$$
\left|B^{\prime}\left(r e^{i \theta}\right)\right| \leq \sum_{n=1}^{\infty} \frac{1-\left|z_{n}\right|^{2}}{\left|1-\bar{z}_{n} r e^{i \theta}\right|^{2}}, \quad\left(r e^{i \theta} \in \mathbb{D}\right)
$$

This work was supported by NSERC (Canada) and FQRNT (Québec). A part of this work was done while the first author was visiting McGill University. He would like to thank this institution for its warm hospitality. 
If (1.1) is the only restriction we put on the zeros of $B$, we can only say that

$$
\begin{aligned}
\int_{0}^{2 \pi}\left|B^{\prime}\left(r e^{i \theta}\right)\right| d \theta & \leq \sum_{n=1}^{\infty}\left(1-\left|z_{n}\right|^{2}\right) \int_{0}^{2 \pi} \frac{d \theta}{\left|1-\bar{z}_{n} r e^{i \theta}\right|^{2}} \\
& =\sum_{n=1}^{\infty}\left(1-\left|z_{n}\right|^{2}\right) \frac{2 \pi}{\left(1-\left|z_{n}\right|^{2} r^{2}\right)} \\
& \leq \frac{4 \pi \sum_{n=1}^{\infty}\left(1-\left|z_{n}\right|\right)}{(1-r)},
\end{aligned}
$$

which implies

$$
\int_{0}^{2 \pi}\left|B^{\prime}\left(r e^{i \theta}\right)\right| d \theta=\frac{o(1)}{1-r}, \quad(r \rightarrow 1)
$$

However, assuming stronger restrictions on the rate of increase of the zeros of $B$ give us more precise estimates about the rate of increase of integral means of $B_{r}^{\prime}$ as $r \rightarrow 1$. The most common restriction is

$$
\sum_{n=1}^{\infty}\left(1-\left|z_{n}\right|\right)^{\alpha}<\infty
$$

for some $\alpha \in(0,1)$. Protas [15] took the first step in this direction by proving the following results.

Let us mention that $H^{p}, 0<p<\infty$, stands for the classical Hardy space equipped with the norm

$$
\|f\|_{p}=\lim _{r \rightarrow 1}\left(\int_{0}^{2 \pi}\left|f\left(r e^{i \theta}\right)\right|^{p} \frac{d \theta}{2 \pi}\right)^{\frac{1}{p}}
$$

and its cousin $A_{\gamma}^{p}, 0<p<\infty$ and $\gamma>-1$, stands for the (weighted) Bergman space equipped with the norm

$$
\|f\|_{p, \gamma}=\left(\int_{0}^{1} \int_{0}^{2 \pi}\left|f\left(r e^{i \theta}\right)\right|^{p} \frac{r\left(1-r^{2}\right)^{\gamma} d r d \theta}{\pi /(1+\gamma)}\right)^{\frac{1}{p}}
$$

THEOREM 1.1 (Protas). If $0<\alpha<\frac{1}{2}$ and the Blaschke sequence $\left(z_{n}\right)_{n \geq 1}$ satisfies (1.4), then $B^{\prime} \in H^{1-\alpha}$.

THEOREM 1.2 (Protas). If $0<\alpha<1$ and the Blaschke sequence $\left(z_{n}\right)_{n \geq 1}$ satisfies (1.4), then $B^{\prime} \in A_{\alpha-1}^{1}$.

Then, Ahern and Clark [1] showed that Theorem 1.1 is sharp in the sense that $B^{\prime}$ need not lie in any $H^{p}$ with $p>1-\alpha$. Later on, they also showed that the condition $\sum_{n=1}^{\infty}\left(1-\left|z_{n}\right|\right)^{1 / 2}<\infty$ is not enough to imply that $B^{\prime} \in H^{1 / 2}$ [2]. At the same time, Linden [12] generalized Theorem 1.1 for higher derivatives of $B$. In the converse direction, Ahern and Clark [1] also obtained the following result. 
THEOREM 1.3 (Ahern-Clark). If $\frac{1}{2}<p<1$, then there is a Blaschke product $B$ with $B^{\prime} \in H^{p}$, and such that its zeros satisfies

$$
\sum_{n=1}^{\infty}\left(1-\left|z_{n}\right|\right)^{\alpha}=\infty
$$

for all $\alpha$ with $0<\alpha<(1-p) / p$.

However, Cohn [3] proved that for interpolating sequences the two conditions are equivalent.

THEOREM $1.4(\mathrm{Cohn})$. Let $0<\alpha<\frac{1}{2}$, and let $\left(z_{n}\right)_{n \geq 1}$ be an interpolating Blaschke sequence. Then, $B^{\prime} \in H^{1-\alpha}$ if and only if $\left(z_{n}\right)_{n \geq 1}$ satisfies (1.4).

Recently, Kutbi [11] showed that under the hypothesis of Theorem 1.1,

$$
\int_{0}^{2 \pi}\left|B^{\prime}\left(r e^{i \theta}\right)\right|^{p} d \theta=\frac{o(1)}{(1-r)^{p+\alpha-1}}, \quad(r \rightarrow 1),
$$

for any $p>1-\alpha$. In particular, for $p=1$, we have

$$
\int_{0}^{2 \pi}\left|B^{\prime}\left(r e^{i \theta}\right)\right| d \theta=\frac{o(1)}{(1-r)^{\alpha}}, \quad(r \rightarrow 1),
$$

which is a refinement of (1.3).

Then, Protas [16] proved that the estimate (1.5) is still valid if $1 / 2<\alpha \leq 1, p \geq \alpha$ and the Blaschke sequence $\left(z_{n}\right)_{n \geq 1}$ satisfies (1.4). Finally, Gotoh [7] got an extension of Protas's results for higher derivatives of $B$.

A Blaschke sequence which satisfies the Carleson condition is called an interpolation, or Carleson, Blaschke sequence [10, p. 200]. Let $I$ be an inner function for the unit disc. In particular, $I$ could be any Blaschke product. Then,

$$
K_{I}:=H^{2} \ominus I H^{2}
$$

is called the model subspace of $H^{2}$ generated by the inner function $I[6,8]$. Cohn [3] obtained the following result about the derivative of functions in $K_{B}$.

THEOREM $1.5(\mathrm{Cohn})$. Let $\left(z_{n}\right)_{n \geq 1}$ be an interpolating Blaschke sequence, and let $p \in(2 / 3,1)$. Then, $B^{\prime} \in H^{p}$ if and only if $f^{\prime} \in H^{2 p /(p+2)}$ for all $f \in K_{B}$.

In this paper, we replace the condition (1.4) by a more general assumption

$$
\sum_{n=1}^{\infty} h\left(1-\left|z_{n}\right|\right)<\infty
$$

where $h$ is a positive continuous function satisfying certain smoothness conditions, and then we generalize all the preceding results. Since our sequence already satisfies the Blaschke condition, (1.6) will provide further information about the rate of increase of the zeros only if $h(t) \geq t$ as $t \rightarrow 0$.

In particular, we are interested in

$$
h(t)=t^{\alpha}(\log 1 / t)^{\alpha_{1}}\left(\log _{2} 1 / t\right)^{\alpha_{2}} \ldots\left(\log _{n} 1 / t\right)^{\alpha_{n}},
$$

where $\alpha \in(0,1), \alpha_{1}, \alpha_{2}, \cdots, \alpha_{n} \in \mathbb{R}$, and $\log _{n}=\log \log \cdots \log (n$ times) [13]. 
In the following, we will use the estimates

$$
\begin{aligned}
\int_{0}^{2 \pi} \frac{d \theta}{\left|1-r e^{i \theta}\right|^{v}} & \asymp \frac{1}{(1-r)^{\nu-1}}, \quad(v>1), \\
\int_{0}^{1} \int_{0}^{2 \pi} \frac{\left(1-\rho^{2}\right)^{\gamma}}{\left|1-r \rho e^{i \theta}\right|^{v}} \rho d \rho d \theta & \asymp \frac{1}{(1-r)^{\nu-\gamma-2}}, \quad(v-2>\gamma>-1),
\end{aligned}
$$

as $r \rightarrow 1^{-}$. See $\left[9\right.$, p. 7]. Both relations can be proved using the fact that $\left|1-r e^{i \theta}\right| \asymp$ $(1-r)+|\theta|$ as $r \rightarrow 1^{-}$.

2. An estimation lemma. In the following we assume that $h$ is a continuous positive function defined on the interval $(0,1)$ with

$$
\lim _{t \rightarrow 0^{+}} h(t)=0 .
$$

Our prototype is the one given in (1.7). The following lemma has simple assumptions and also a very simple proof. However, it has many interesting applications in the rest of the paper.

LEMMA 2.1. Let $\left(r_{n}\right)_{n \geq 1}$ be a sequence in the interval $(0,1)$ such that

$$
\sum_{n=1}^{\infty} h\left(1-r_{n}\right)<\infty
$$

Let $p, q>0$ be such that $h(t) / t^{p}$ is decreasing and $h(t) / t^{p-q}$ is increasing on $(0,1)$. Then,

$$
\sum_{n=1}^{\infty} \frac{\left(1-r_{n}\right)^{p}}{\left(1-r r_{n}\right)^{q}}=\frac{O(1)}{(1-r)^{q-p} h(1-r)}
$$

as $r \rightarrow 1^{-}$. Moreover, if

$$
\lim _{t \rightarrow 0^{+}} \frac{h(t)}{t^{p-q}}=0
$$

then

$$
\sum_{n=1}^{\infty} \frac{\left(1-r_{n}\right)^{p}}{\left(1-r r_{n}\right)^{q}}=\frac{o(1)}{(1-r)^{q-p} h(1-r)}
$$

Proof. We have

$$
\frac{\left(1-r_{n}\right)^{p}}{\left(1-r r_{n}\right)^{q}}=\left(\frac{\left(1-r_{n}\right)^{p}}{h\left(1-r_{n}\right)} \frac{h\left(1-r r_{n}\right)}{\left(1-r r_{n}\right)^{p}}\right)\left(\frac{h\left(1-r_{n}\right)}{\left(1-r r_{n}\right)^{q-p} h\left(1-r r_{n}\right)}\right) .
$$

By assumption

$$
\frac{h\left(1-r r_{n}\right)}{\left(1-r r_{n}\right)^{p}} \leq \frac{h\left(1-r_{n}\right)}{\left(1-r_{n}\right)^{p}}
$$

and

$$
\left(1-r r_{n}\right)^{q-p} h\left(1-r r_{n}\right) \geq(1-r)^{q-p} h(1-r) .
$$


Thus, for any $n \geq 1$,

$$
\frac{\left(1-r_{n}\right)^{p}}{\left(1-r r_{n}\right)^{q}} \leq \frac{h\left(1-r_{n}\right)}{(1-r)^{q-p} h(1-r)} .
$$

Given $\varepsilon>0$, fix $N$ such that

$$
\sum_{n=N+1}^{\infty} h\left(1-r_{n}\right)<\varepsilon
$$

Hence, by (2.1),

$$
\begin{aligned}
\sum_{n=1}^{\infty} \frac{\left(1-r_{n}\right)^{p}}{\left(1-r r_{n}\right)^{q}} & =\sum_{n=1}^{N} \frac{\left(1-r_{n}\right)^{p}}{\left(1-r r_{n}\right)^{q}}+\sum_{n=N+1}^{\infty} \frac{\left(1-r_{n}\right)^{p}}{\left(1-r r_{n}\right)^{q}} \\
& \leq \sum_{n=1}^{N}\left(1-r_{n}\right)^{p-q}+\frac{\sum_{n=N+1}^{\infty} h\left(1-r_{n}\right)}{(1-r)^{q-p} h(1-r)} \\
& \leq C_{N}+\frac{\varepsilon}{(1-r)^{q-p} h(1-r)}
\end{aligned}
$$

where $C_{N}$ is independent of $r$. This inequality implies both assertions of the Lemma.

The Lemma is still valid if instead of "decreasing" and "increasing", we assume that our functions are respectively "boundedly decreasing" and "boundedly increasing". We say that $\varphi$ is boundedly increasing if there is a constant $C>0$ such that $\varphi(x) \leq C \varphi(y)$ whenever $x \leq y$. Similarly, $\varphi$ is boundedly decreasing if there is a constant $C>0$ such that $\varphi(x) \geq C \varphi(y)$ whenever $x \leq y$.

3. $H^{p}$ means of the first derivative. In this section we apply Lemma 2.1 to obtain a general estimate for the integral means of the first derivative of a Blaschke product. Special cases of the following theorem generalize Protas and Kutbi's results.

THEOREM 3.1. Let $B$ be the Blaschke product formed with zeros $z_{n}=r_{n} e^{i \theta_{n}}, n \geq 1$, satisfying

$$
\sum_{n=1}^{\infty} h\left(1-r_{n}\right)<\infty
$$

for a positive continuous function $h$. Suppose that there is $q \in(1 / 2,1]$ such that $h(t) / t^{q}$ is decreasing and $h(t) / t^{1-q}$ is increasing on $(0,1)$. Then, for any $p \geq q$,

$$
\int_{0}^{2 \pi}\left|B^{\prime}\left(r e^{i \theta}\right)\right|^{p} d \theta=\frac{O(1)}{(1-r)^{p-1} h(1-r)}, \quad(r \rightarrow 1) .
$$

Moreover, if $\lim _{t \rightarrow 0} h(t) / t^{1-q}=0$, then $O(1)$ can be replaced by $o(1)$. 
Proof. Since $q \leq 1,(1.2)$ implies

$$
\left|B^{\prime}\left(r e^{i \theta}\right)\right|^{q} \leq \sum_{n=1}^{\infty} \frac{\left(1-r_{n}^{2}\right)^{q}}{\left|1-r r_{n} e^{i\left(\theta-\theta_{n}\right)}\right|^{2 q}} .
$$

Hence

$$
\int_{0}^{2 \pi}\left|B^{\prime}\left(r e^{i \theta}\right)\right|^{q} d \theta \leq C \sum_{n=1}^{\infty} \frac{\left(1-r_{n}\right)^{q}}{\left(1-r r_{n}\right)^{2 q-1}} .
$$

(Here we used $2 q>1$.) Therefore, by Lemma 2.1,

$$
\int_{0}^{2 \pi}\left|B^{\prime}\left(r e^{i \theta}\right)\right|^{q} d \theta \leq \frac{C}{(1-r)^{q-1} h(1-r)} .
$$

Now recall that any function $f$ in $H^{\infty}$ is in the Bloch space $\mathcal{B}[4, \mathrm{p} .44]$, that is

$$
\sup _{z \in \mathbb{D}}\left(1-|z|^{2}\right)\left|f^{\prime}(z)\right|<+\infty .
$$

Hence, for any $p \geq q$,

$$
\int_{0}^{2 \pi}\left|B^{\prime}\left(r e^{i \theta}\right)\right|^{p} d \theta \leq \frac{1}{(1-r)^{p-q}} \int_{0}^{2 \pi}\left|B^{\prime}\left(r e^{i \theta}\right)\right|^{q} d \theta \leq \frac{C}{(1-r)^{p-1} h(1-r)} .
$$

Finally, as $r \rightarrow 1$, Lemma 2.1 also assures that $C$ can be replaced by any small positive constant if $\lim _{t \rightarrow 0} h(t) / t^{1-q}=0$.

Now, we can apply Theorem 3.1 for the special function defined in (1.7).

Case 1. If

$$
\sum_{n=1}^{\infty}\left(1-r_{n}\right)^{\alpha}\left(\log \frac{1}{1-r_{n}}\right)^{\alpha_{1}} \cdots\left(\log _{m} \frac{1}{1-r_{n}}\right)^{\alpha_{m}}<\infty
$$

then, for any

$$
p>\max \{\alpha, 1-\alpha\}
$$

we have

$$
\int_{0}^{2 \pi}\left|B^{\prime}\left(r e^{i \theta}\right)\right|^{p} d \theta=\frac{o(1)}{(1-r)^{\alpha+p-1}\left(\log \frac{1}{1-r}\right)^{\alpha_{1}} \cdots\left(\log _{m} \frac{1}{1-r}\right)^{\alpha_{m}}}, \quad(r \rightarrow 1) .
$$

In particular, if

$$
\sum_{n=1}^{\infty}\left(1-r_{n}\right)^{\alpha}<\infty
$$

with $\alpha \in(0,1 / 2)$, then, for any $p>1-\alpha$,

$$
\int_{0}^{2 \pi}\left|B^{\prime}\left(r e^{i \theta}\right)\right|^{p} d \theta=\frac{o(1)}{(1-r)^{p+\alpha-1}}, \quad(r \rightarrow 1),
$$


which is Kutbi's result. Moreover, if $\alpha \in[1 / 2,1)$, the last estimate still holds for any $p>\alpha$, which is Protas's result [16].

Case 2. If

$$
\sum_{n=1}^{\infty}\left(1-r_{n}\right)^{\alpha}\left(\log _{k} \frac{1}{1-r_{n}}\right)^{\alpha_{k}} \cdots\left(\log _{m} \frac{1}{1-r_{n}}\right)^{\alpha_{m}}<\infty
$$

with $\alpha \in(0,1 / 2), \alpha_{k}<0$ and $\alpha_{k+1}, \cdots, \alpha_{m} \in \mathbb{R}$, then,

$$
\int_{0}^{2 \pi}\left|B^{\prime}\left(r e^{i \theta}\right)\right|^{1-\alpha} d \theta=\frac{o(1)}{\left(\log _{k} \frac{1}{1-r}\right)^{\alpha_{k}} \cdots\left(\log _{m} \frac{1}{1-r}\right)^{\alpha_{m}}}, \quad(r \rightarrow 1) .
$$

But, if

$$
\sum_{n=1}^{\infty}\left(1-r_{n}\right)^{\alpha}<\infty
$$

with $\alpha \in(0,1 / 2)$, then

$$
\int_{0}^{2 \pi}\left|B^{\prime}\left(r e^{i \theta}\right)\right|^{1-\alpha} d \theta=O(1), \quad(r \rightarrow 1),
$$

i.e. $B^{\prime} \in H^{1-\alpha}$, which is Protas' result [15].

Case 3. If

$$
\sum_{n=1}^{\infty}\left(1-r_{n}\right)^{\alpha}\left(\log _{k} \frac{1}{1-r_{n}}\right)^{\alpha_{k}} \cdots\left(\log _{m} \frac{1}{1-r_{n}}\right)^{\alpha_{m}}<\infty
$$

with $\alpha \in(1 / 2,1), \alpha_{k}>0$ and $\alpha_{k+1}, \cdots, \alpha_{n} \in \mathbb{R}$, then,

$$
\int_{0}^{2 \pi}\left|B^{\prime}\left(r e^{i \theta}\right)\right|^{\alpha} d \theta=\frac{o(1)}{(1-r)^{2 \alpha-1}\left(\log \frac{1}{1-r}\right)^{\alpha_{1}} \cdots\left(\log _{m} \frac{1}{1-r}\right)^{\alpha_{m}}}, \quad(r \rightarrow 1) .
$$

However, if

$$
\sum_{n=1}^{\infty}\left(1-r_{n}\right)^{\alpha}<\infty
$$

with $\alpha \in(1 / 2,1)$, then we still have

$$
\int_{0}^{2 \pi}\left|B^{\prime}\left(r e^{i \theta}\right)\right|^{\alpha} d \theta=\frac{o(1)}{(1-r)^{2 \alpha-1}}, \quad(r \rightarrow 1) .
$$

4. $H^{p}$ means of higher derivatives. Straightforward calculation leads to

$$
\int_{0}^{2 \pi}\left|B^{(\ell)}\left(r e^{i \theta}\right)\right|^{p} d \theta \leq C(p, \ell) \sum_{n=1}^{\infty} \frac{\left(1-r_{n}\right)^{p}}{\left(1-r r_{n}\right)^{(\ell+1) p-1}}, \quad\left(\frac{1}{\ell+1}<p \leq \frac{1}{\ell}\right),
$$


which is a generalization of (3.1). This observation along with Lemma 2.1 enable us to generalize the results of the preceding section for higher derivatives of a Blaschke product. The proof is similar to that of Theorem 3.1.

THEOREM 4.1. Let $B$ be the Blaschke product formed with zeros $z_{n}=r_{n} e^{i \theta_{n}}, n \geq 1$, satisfying

$$
\sum_{n=1}^{\infty} h\left(1-r_{n}\right)<\infty
$$

for a positive continuous function $h$. Suppose that there is $q \in(1 /(\ell+1), 1 / \ell]$ such that $h(t) / t^{q}$ is decreasing and $h(t) / t^{1-\ell q}$ is increasing on $(0,1)$. Then, for any $p \geq q$,

$$
\int_{0}^{2 \pi}\left|B^{(\ell)}\left(r e^{i \theta}\right)\right|^{p} d \theta=\frac{O(1)}{(1-r)^{\ell p-1} h(1-r)}, \quad(r \rightarrow 1) .
$$

Moreover, if $\lim _{t \rightarrow 0} h(t) / t^{1-\ell q}=0$, then $O(1)$ can be replaced by $o(1)$.

Now, we can apply Theorem 4.1 for the special function defined in (1.7).

Case 1. If

$$
\sum_{n=1}^{\infty}\left(1-r_{n}\right)^{\alpha}\left(\log \frac{1}{1-r_{n}}\right)^{\alpha_{1}} \cdots\left(\log _{m} \frac{1}{1-r_{n}}\right)^{\alpha_{m}}<\infty
$$

then, for any

$$
p>\max \{\alpha,(1-\alpha) / \ell\}
$$

we have

$$
\int_{0}^{2 \pi}\left|B^{(\ell)}\left(r e^{i \theta}\right)\right|^{p} d \theta=\frac{o(1)}{(1-r)^{\alpha+\ell p-1}\left(\log \frac{1}{1-r}\right)^{\alpha_{1}} \cdots\left(\log _{m} \frac{1}{1-r}\right)^{\alpha_{m}}}, \quad(r \rightarrow 1) .
$$

In particular, if

$$
\sum_{n=1}^{\infty}\left(1-r_{n}\right)^{\alpha}<\infty
$$

with $\alpha \in(0,1 /(\ell+1))$, then, for any $p>(1-\alpha) / \ell$,

$$
\int_{0}^{2 \pi}\left|B^{(\ell)}\left(r e^{i \theta}\right)\right|^{p} d \theta=\frac{o(1)}{(1-r)^{\ell p+\alpha-1}}, \quad(r \rightarrow 1),
$$

which is Kutbi's result. Moreover, if $\alpha \in[1 /(\ell+1), 1)$, the last estimate still holds for any $p>\alpha$, which is Gotoh's result [7] .

Case 2. If

$$
\sum_{n=1}^{\infty}\left(1-r_{n}\right)^{\alpha}\left(\log _{k} \frac{1}{1-r_{n}}\right)^{\alpha_{k}} \cdots\left(\log _{m} \frac{1}{1-r_{n}}\right)^{\alpha_{m}}<\infty
$$


with $\alpha \in(0,1 /(1+\ell)), \alpha_{k}<0$ and $\alpha_{k+1}, \cdots, \alpha_{n} \in \mathbb{R}$, then,

$$
\int_{0}^{2 \pi}\left|B^{(\ell)}\left(r e^{i \theta}\right)\right|^{(1-\alpha) / \ell} d \theta=\frac{o(1)}{\left(\log _{k} \frac{1}{1-r}\right)^{\alpha_{k}} \cdots\left(\log _{m} \frac{1}{1-r}\right)^{\alpha_{m}}}, \quad(r \rightarrow 1) .
$$

But, if

$$
\sum_{n=1}^{\infty}\left(1-r_{n}\right)^{\alpha}<+\infty
$$

with $\alpha \in(0,1 /(1+\ell))$, then

$$
\int_{0}^{2 \pi}\left|B^{(\ell)}\left(r e^{i \theta}\right)\right|^{(1-\alpha) / \ell} d \theta=O(1), \quad(r \rightarrow 1)
$$

i.e. $B^{(\ell)} \in H^{(1-\alpha) / \ell}$ which is Linden's result [12].

Case 3. If

$$
\sum_{n=1}^{\infty}\left(1-r_{n}\right)^{\alpha}\left(\log _{k} \frac{1}{1-r_{n}}\right)^{\alpha_{k}} \cdots\left(\log _{m} \frac{1}{1-r_{n}}\right)^{\alpha_{m}}<\infty
$$

with $\alpha \in(1 /(1+\ell), 1), \alpha_{k}>0$ and $\alpha_{k+1}, \cdots, \alpha_{n} \in \mathbb{R}$, then,

$$
\int_{0}^{2 \pi}\left|B^{(\ell)}\left(r e^{i \theta}\right)\right|^{\alpha} d \theta=\frac{o(1)}{(1-r)^{(\ell+1) \alpha-1}\left(\log _{k} \frac{1}{1-r}\right)^{\alpha_{k}} \cdots\left(\log _{m} \frac{1}{1-r}\right)^{\alpha_{m}}}, \quad(r \rightarrow 1) .
$$

However, if

$$
\sum_{n=1}^{\infty}\left(1-r_{n}\right)^{\alpha}<\infty
$$

with $\alpha \in(1 /(\ell+1), 1)$, then we still have

$$
\int_{0}^{2 \pi}\left|B^{(\ell)}\left(r e^{i \theta}\right)\right|^{\alpha} d \theta=\frac{o(1)}{(1-r)^{(\ell+1) \alpha-1}}, \quad(r \rightarrow 1) .
$$

5. $A_{\gamma}^{p}$ means of the first derivative. In this section we apply Lemma 2.1 to obtain a general estimate for the integral means of the first derivative of a Blaschke product. Special cases of the following theorem generalize Protas's results [15].

THEOREM 5.1. Let B be the Blaschke product formed with zeros $z_{n}=r_{n} e^{i \theta_{n}}$ satisfying

$$
\sum_{n=1}^{\infty} h\left(1-r_{n}\right)<\infty
$$

for a positive continuous function $h$. Let $\gamma \in(-1,0)$. Suppose that there is $q \in(1+$ $\gamma / 2,1]$ such that $h(t) / t^{q}$ is decreasing and $h(t) / t^{2+\gamma-q}$ is increasing on $(0,1)$. Then, for 
any $p \geq q$,

$$
\int_{0}^{1} \int_{0}^{2 \pi}\left|B^{\prime}\left(r \rho e^{i \theta}\right)\right|^{p} \quad \rho\left(1-\rho^{2}\right)^{\gamma} d \rho d \theta=\frac{O(1)}{(1-r)^{p-\gamma-2} h(1-r)}, \quad(r \rightarrow 1) .
$$

Moreover, if $\lim _{t \rightarrow 0} h(t) / t^{2+\gamma-q}=0$, then $O(1)$ can be replaced by $o(1)$.

Proof. We saw that

$$
\left|B^{\prime}\left(r \rho e^{i \theta}\right)\right|^{q} \leq \sum_{n=1}^{\infty} \frac{\left(1-r_{n}^{2}\right)^{q}}{\left|1-r r_{n} \rho e^{i\left(\theta-\theta_{n}\right)}\right|^{2 q}} .
$$

Hence

$$
\int_{0}^{1} \int_{0}^{2 \pi}\left|B^{\prime}\left(r \rho e^{i \theta}\right)\right|^{q} \rho\left(1-\rho^{2}\right)^{\gamma} d \rho d \theta \leq C \sum_{n=1}^{\infty} \frac{\left(1-r_{n}\right)^{q}}{\left(1-r r_{n}\right)^{2 q-\gamma-2}} .
$$

(Here we used $2 q-\gamma-2>0$.) Therefore, by Lemma 2.1,

$$
\int_{0}^{1} \int_{0}^{2 \pi}\left|B^{\prime}\left(r \rho e^{i \theta}\right)\right|^{q} \rho\left(1-\rho^{2}\right)^{\gamma} d \rho d \theta \leq \frac{C}{(1-r)^{q-\gamma-2} h(1-r)} .
$$

Hence, for any $p \geq q$,

$$
\begin{aligned}
\int_{0}^{1} \int_{0}^{2 \pi}\left|B^{\prime}\left(r \rho e^{i \theta}\right)\right|^{p} \rho\left(1-\rho^{2}\right)^{\gamma} d \rho d \theta & \leq \frac{1}{(1-r)^{p-q}} \int_{0}^{1} \int_{0}^{2 \pi}\left|B^{\prime}\left(r \rho e^{i \theta}\right)\right|^{q} \rho\left(1-\rho^{2}\right)^{\gamma} d \rho d \theta \\
& \leq \frac{C}{(1-r)^{p-\gamma-2} h(1-r)} .
\end{aligned}
$$

Finally, as $r \rightarrow 1$, Lemma 2.1 also assures that $C$ can be replaced by any small positive constant if $\lim _{t \rightarrow 0} h(t) / t^{2+\gamma-q}=0$.

Now, we can apply Theorem 5.1 for the special function defined in (1.7).

Case 1. If

$$
\sum_{n=1}^{\infty}\left(1-r_{n}\right)^{\alpha}\left(\log \frac{1}{1-r_{n}}\right)^{\alpha_{1}} \cdots\left(\log _{m} \frac{1}{1-r_{n}}\right)^{\alpha_{m}}<\infty
$$

and if $\gamma \in(-1, \alpha-1)$, then, for any

$$
p>\max \{\alpha, 2+\gamma-\alpha, 1+\gamma / 2\},
$$

we have

$$
\int_{0}^{1} \int_{0}^{2 \pi}\left|B^{\prime}\left(r \rho e^{i \theta}\right)\right|^{p} \rho\left(1-\rho^{2}\right)^{\gamma} d \rho d \theta=\frac{o(1)}{(1-r)^{\alpha+p-\gamma-2}\left(\log \frac{1}{1-r}\right)^{\alpha_{1}} \cdots\left(\log _{m} \frac{1}{1-r}\right)^{\alpha_{m}}},
$$

as $(r \rightarrow 1)$. In particular, if

$$
\sum_{n=1}^{\infty}\left(1-r_{n}\right)^{\alpha}<\infty
$$


then

$$
\int_{0}^{1} \int_{0}^{2 \pi}\left|B^{\prime}\left(r \rho e^{i \theta}\right)\right|^{p} \rho\left(1-\rho^{2}\right)^{\gamma} d \rho d \theta=\frac{o(1)}{(1-r)^{p+\alpha-\gamma-2}} .
$$

Case 2. If

$$
\sum_{n=1}^{\infty}\left(1-r_{n}\right)^{\alpha}\left(\log _{k} \frac{1}{1-r_{n}}\right)^{\alpha_{k}} \cdots\left(\log _{m} \frac{1}{1-r_{n}}\right)^{\alpha_{m}}<\infty
$$

with $\alpha_{k}<0$, then, for any $p \geq 1$,

$$
\begin{aligned}
\int_{0}^{1} \int_{0}^{2 \pi}\left|B^{\prime}\left(r \rho e^{i \theta}\right)\right|^{p} \rho\left(1-\rho^{2}\right)^{\alpha-1} d \rho d \theta= & \frac{o(1)}{(1-r)^{p-1}\left(\log _{k} \frac{1}{1-r}\right)^{\alpha_{k}} \cdots\left(\log _{m} \frac{1}{1-r}\right)^{\alpha_{m}},} \\
& \times(r \rightarrow 1) .
\end{aligned}
$$

Case 3. If

$$
\sum_{n=1}^{\infty}\left(1-r_{n}\right)^{\alpha}<\infty
$$

then, for any $p \geq 1$,

$$
\int_{0}^{1} \int_{0}^{2 \pi}\left|B^{\prime}\left(r \rho e^{i \theta}\right)\right|^{p} \rho\left(1-\rho^{2}\right)^{\alpha-1} d \rho d \theta=\frac{O(1)}{(1-r)^{p-1}}, \quad(r \rightarrow 1) .
$$

In particular, for $p=1$,

$$
\int_{0}^{1} \int_{0}^{2 \pi}\left|B^{\prime}\left(r \rho e^{i \theta}\right)\right| \rho\left(1-\rho^{2}\right)^{\alpha-1} d \rho d \theta=O(1), \quad(r \rightarrow 1) .
$$

which is the Protas' result [15].

Some other cases can also be considered here. But, since they are an immediate consequence of Theorem 5.1, we do not proceed further. Moreover, using similar techniques, one can obtain estimates for the $A_{\gamma}^{p}$ means of the higher derivatives for a Blaschke product satisfying the hypothesis of Theorem 5.1.

6. Interpolating Blaschke products. Cohn's theorems 1.4 and 1.5 imply that if $z_{n}=r_{n} e^{i \theta_{n}}, n \geq 1$, is a Carleson sequence satisfying

$$
\sum_{n=1}^{\infty}\left(1-r_{n}\right)^{1-p}<\infty
$$

for some $p \in(2 / 3,1)$, then $f^{\prime} \in H^{2 p /(p+2)}$ for all $f \in K_{B}$. The following result generalizes this fact.

THEOREM 6.1. Let $z_{n}=r_{n} e^{i \theta_{n}}, n \geq 1$, be a Carleson sequence satisfying

$$
\sum_{n=1}^{\infty} h\left(1-r_{n}\right)<\infty
$$


for a positive continuous function $h$. Let $B$ be the Blaschke product formed with zeros $z_{n}$, $n \geq 1$. Suppose that there is $p \in(2 / 3,1)$ such that $h(t) / t^{p / 2}$ is decreasing and $h(t) / t^{1-p}$ is increasing on $(0,1)$. Then, for all $f \in K_{B}$, we have

$$
\left(\int_{0}^{2 \pi}\left|f^{\prime}\left(r e^{i \theta}\right)\right|^{\sigma} d \theta\right)^{1 / \sigma} \leq \frac{C\|f\|_{2}}{\left((1-r)^{p-1} h(1-r)\right)^{1 / p}}, \quad(r \rightarrow 1)
$$

with $\sigma=2 p /(p+2)$ and $C$ an absolute constant.

Proof. Since $\left(z_{n}\right)_{n \geq 1}$ is a Carleson sequence, we know that the functions

$$
f_{n}(z):=\frac{\left(1-r_{n}\right)^{1 / 2}}{1-\bar{z}_{n} z}, \quad(n \geq 1),
$$

form a Riesz basis of $K_{B}$ (see [14] for instance). Now, let $f=\sum_{n=1}^{N} \beta_{n} f_{n}, \beta_{n} \in \mathbb{C}$. Then

$$
f^{\prime}(z)=\sum_{n=1}^{N} \frac{\bar{z}_{n} \beta_{n}\left(1-r_{n}\right)^{1 / 2}}{\left(1-\bar{z}_{n} z\right)^{2}}
$$

and thus we get

$$
\left|f^{\prime}(z)\right| \leq \sum_{n=1}^{N} \frac{\left|\beta_{n}\right|\left(1-r_{n}\right)^{1 / 2}}{\left|1-\bar{z}_{n} z\right|^{2}}
$$

Since $p \in(2 / 3,1)$, we have $\sigma \in(1 / 2,1)$ and we can write

$$
\left|f^{\prime}(z)\right|^{\sigma} \leq \sum_{n=1}^{N} \frac{\left|\beta_{n}\right|^{\sigma}\left(1-r_{n}\right)^{\sigma / 2}}{\left|1-\bar{z}_{n} z\right|^{2 \sigma}}
$$

Therefore,

$$
\begin{aligned}
\int_{0}^{2 \pi}\left|f^{\prime}\left(r e^{i \theta}\right)\right|^{\sigma} d \theta & \leq \sum_{n=1}^{N}\left|\beta_{n}\right|^{\sigma}\left(1-r_{n}\right)^{\sigma / 2} \int_{0}^{2 \pi} \frac{d \theta}{\left|1-\bar{z}_{n} r e^{i \theta}\right|^{2 \sigma}} \\
& \leq c \sum_{n=1}^{N}\left|\beta_{n}\right|^{\sigma} \frac{\left(1-r_{n}\right)^{\sigma / 2}}{\left(1-r r_{n}\right)^{2 \sigma-1}} .
\end{aligned}
$$

Let $p^{\prime}=2 / \sigma$ and let $q^{\prime}$ be its conjugate exponent. Then Hölder's inequality implies that

$$
\int_{0}^{2 \pi}\left|f^{\prime}\left(r e^{i \theta}\right)\right|^{\sigma} d \theta \leq c\left(\sum_{n=1}^{N}\left|\beta_{n}\right|^{2}\right)^{1 / p^{\prime}}\left(\sum_{n=1}^{N} \frac{\left(1-r_{n}\right)^{\sigma q^{\prime} / 2}}{\left(1-r r_{n}\right)^{(2 \sigma-1) q^{\prime}}}\right)^{1 / q^{\prime}}
$$

But since $\left(f_{n}\right)_{n \geq 1}$ forms a Riesz basis of $K_{B}$, there exists a constant $c_{1}>0$ such that

$$
\sum_{n=1}^{N}\left|\beta_{n}\right|^{2} \leq c_{1}\|f\|_{2}^{2}
$$


whence

$$
\int_{0}^{2 \pi}\left|f^{\prime}\left(r e^{i \theta}\right)\right|^{\sigma} d \theta \leq c_{2}\|f\|_{2}^{\sigma}\left(\sum_{n=1}^{N} \frac{\left(1-r_{n}\right)^{\sigma q^{\prime} / 2}}{\left(1-r r_{n}\right)^{(2 \sigma-1) q^{\prime}}}\right)^{1 / q^{\prime}} .
$$

Now easy computations show that $q^{\prime}=\frac{p+2}{2}, \sigma q^{\prime}=p,(2 \sigma-1) q^{\prime}=3 p / 2-1$ and therefore, by Lemma 2.1, we have

$$
\sum_{n=1}^{N} \frac{\left(1-r_{n}\right)^{\sigma q^{\prime} / 2}}{\left(1-r r_{n}\right)^{(2 \sigma-1) q^{\prime}}} \leq \frac{C}{(1-r)^{p-1} h(1-r)}
$$

where $C$ is a constant independent of $N$. We deduce that

$$
\int_{0}^{2 \pi}\left|f^{\prime}\left(r e^{i \theta}\right)\right|^{\sigma} d \theta \leq \frac{c_{3}\|f\|_{2}^{\sigma}}{\left((1-r)^{p-1} h(1-r)\right)^{1 / q^{\prime}}} .
$$

Since $1 / \sigma q^{\prime}=1 / p$, and using a density argument, we get that for all $f \in K_{B}$,

$$
\left(\int_{0}^{2 \pi}\left|f^{\prime}\left(r e^{i \theta}\right)\right|^{\sigma} d \theta\right)^{1 / \sigma} \leq \frac{c_{3}^{1 / \sigma}\|f\|_{2}}{\left((1-r)^{p-1} h(1-r)\right)^{1 / p}} .
$$

Now, we can apply Theorem 6.1 for the special function defined in (1.7).

Case 1. If $z_{n}=r_{n} e^{i \theta_{n}}, n \geq 1$, is a Carleson sequence satisfying

$$
\sum_{n=1}^{\infty}\left(1-r_{n}\right)^{\alpha}\left(\log \frac{1}{1-r_{n}}\right)^{\alpha_{1}} \cdots\left(\log _{m} \frac{1}{1-r_{n}}\right)^{\alpha_{m}}<\infty
$$

with $p \in(2 / 3,1), 1-p<\alpha<p / 2$, and $\alpha_{1}, \ldots, \alpha_{m} \in \mathbb{R}$, then, for all $f \in K_{B}$, we have

$$
\left(\int_{0}^{2 \pi}\left|f^{\prime}\left(r e^{i \theta}\right)\right|^{\sigma} d \theta\right)^{1 / \sigma} \leq \frac{C\|f\|_{2}}{\left((1-r)^{\alpha+p-1}\left(\log \frac{1}{1-r}\right)^{\alpha_{1}} \cdots\left(\log _{m} \frac{1}{1-r}\right)^{\alpha_{m}}\right)^{1 / p}},
$$

with $\sigma=2 p /(p+2)$ and $C$ an absolute constant.

Case 2. If $z_{n}=r_{n} e^{i \theta_{n}}, n \geq 1$, is a Carleson sequence satisfying

$$
\sum_{n=1}^{\infty}\left(1-r_{n}\right)^{1-p}\left(\log _{k} \frac{1}{1-r_{n}}\right)^{\alpha_{k}} \cdots\left(\log _{m} \frac{1}{1-r_{n}}\right)^{\alpha_{m}}<\infty,
$$

with $p \in(2 / 3,1), k \geq 1, \alpha_{k}, \alpha_{k+1}, \ldots, \alpha_{m} \in \mathbb{R}$, and $\alpha_{k}<0$, then, for all $f \in K_{B}$, we have

$$
\left(\int_{0}^{2 \pi}\left|f^{\prime}\left(r e^{i \theta}\right)\right|^{\sigma} d \theta\right)^{1 / \sigma} \leq \frac{C\|f\|_{2}}{\left(\left(\log _{k} \frac{1}{1-r}\right)^{\alpha_{k}} \cdots\left(\log _{m} \frac{1}{1-r}\right)^{\alpha_{m}}\right)^{1 / p}}, \quad(r \rightarrow 1),
$$

with $\sigma=2 p /(p+2)$ and $C$ an absolute constant. However, if

$$
\sum_{n=1}^{\infty}\left(1-r_{n}\right)^{1-p}<\infty
$$


then we still have

$$
\left(\int_{0}^{2 \pi}\left|f^{\prime}\left(r e^{i \theta}\right)\right|^{\sigma} d \theta\right)^{1 / \sigma} \leq C\|f\|_{2}, \quad(r \rightarrow 1)
$$

i.e. $f^{\prime} \in H^{2 p /(p+2)}$, for any $f \in K_{B}$, which is Cohn's result.

Case 3. If $z_{n}=r_{n} e^{i \theta_{n}}, n \geq 1$, is a Carleson sequence satisfying

$$
\sum_{n=1}^{\infty}\left(1-r_{n}\right)^{p / 2}\left(\log _{k} \frac{1}{1-r_{n}}\right)^{\alpha_{k}} \ldots\left(\log _{m} \frac{1}{1-r_{n}}\right)^{\alpha_{m}}<\infty
$$

with $p \in(2 / 3,1), k \geq 1, \alpha_{k}, \alpha_{k+1}, \ldots, \alpha_{m} \in \mathbb{R}$, and $\alpha_{k}>0$, then, for all $f \in K_{B}$, we have

$$
\left(\int_{0}^{2 \pi}\left|f^{\prime}\left(r e^{i \theta}\right)\right|^{\sigma} d \theta\right)^{1 / \sigma} \leq \frac{C\|f\|_{2}}{\left((1-r)^{3 p / 2-1}\left(\log _{k} \frac{1}{1-r}\right)^{\alpha_{k}} \cdots\left(\log _{m} \frac{1}{1-r}\right)^{\alpha_{m}}\right)^{1 / p}},
$$

with $\sigma=2 p /(p+2)$ and $C$ an absolute constant. However, if

$$
\sum_{n=1}^{\infty}\left(1-r_{n}\right)^{p / 2}<\infty
$$

then we still have

$$
\left(\int_{0}^{2 \pi}\left|f^{\prime}\left(r e^{i \theta}\right)\right|^{\sigma} d \theta\right)^{1 / \sigma} \leq \frac{C\|f\|_{2}}{(1-r)^{(3 p-2) / 2 p}} .
$$

Using similar techniques we can obtain some results about the $A_{\gamma}^{p}$ means of the derivatives of function in the model subspaces of $H^{2}$.

THEOREM 6.2. Let $z_{n}=r_{n} e^{i \theta_{n}}, n \geq 1$, be a Carleson sequence satisfying

$$
\sum_{n=1}^{\infty} h\left(1-r_{n}\right)<\infty
$$

for a positive continuous function $h$, and let $B$ be the Blaschke product formed with zeros $z_{n}, n \geq 1$. Let $p \in(2 / 3,1), \sigma=2 p /(p+2)$ and $-1<\gamma<2 \sigma-2$ such that $h(t) / t^{p / 2}$ is decreasing and $h(t) / t^{(1-p)+(1+\gamma)(1+p / 2)}$ is increasing on $(0,1)$. Then, for all $f \in K_{B}$, we have

$$
\left(\int_{0}^{1} \int_{0}^{2 \pi}\left|f^{\prime}\left(r \rho e^{i \theta}\right)\right|^{\sigma} \rho\left(1-\rho^{2}\right)^{\gamma} d \rho d \theta\right)^{1 / \sigma} \leq \frac{C\|f\|_{2}}{\left((1-r)^{-(1-p)-(1+\gamma)(1+p / 2)} h(1-r)\right)^{1 / p}}
$$

as $r \rightarrow 1^{-}$.

Proof. The beginning of the proof is as that of Theorem 6.1 until

$$
\left|f^{\prime}(z)\right|^{\sigma} \leq \sum_{n=1}^{N} \frac{\left|\beta_{n}\right|^{\sigma}\left(1-r_{n}\right)^{\sigma / 2}}{\left|1-\bar{z}_{n} z\right|^{2 \sigma}}
$$


Therefore, by Hölder's inequality (with $p^{\prime}=2 / \sigma$ and $q^{\prime}$ its conjugate exponent) and by Lemma 2.1, we have

$$
\begin{aligned}
\int_{0}^{1} \int_{0}^{2 \pi}\left|f^{\prime}\left(r \rho e^{i \theta}\right)\right|^{\sigma} \rho\left(1-\rho^{2}\right)^{\gamma} d \rho d \theta & \leq \sum_{n=1}^{N}\left|\beta_{n}\right|^{\sigma}\left(1-r_{n}\right)^{\sigma / 2} \int_{0}^{1} \int_{0}^{2 \pi} \frac{\rho\left(1-\rho^{2}\right)^{\gamma} d \rho d \theta}{\left|1-\bar{z}_{n} r \rho e^{i \theta}\right|^{2 \sigma}} \\
& \leq c \sum_{n=1}^{N}\left|\beta_{n}\right|^{\sigma} \frac{\left(1-r_{n}\right)^{\sigma / 2}}{\left(1-r r_{n}\right)^{2 \sigma-\gamma-2}} \\
& \leq c\left(\sum_{n=1}^{N}\left|\beta_{n}\right|^{2}\right)^{1 / p^{\prime}}\left(\sum_{n=1}^{N} \frac{\left(1-r_{n}\right)^{\sigma q^{\prime} / 2}}{\left(1-r r_{n}\right)^{(2 \sigma-\gamma-2) q^{\prime}}}\right)^{1 / q^{\prime}} \\
& \leq c^{\prime}\|f\|_{2}^{\sigma}\left(\sum_{n=1}^{N} \frac{\left(1-r_{n}\right)^{\sigma q^{\prime} / 2}}{\left(1-r r_{n}\right)^{(2 \sigma-\gamma-2) q^{\prime}}}\right)^{1 / q^{\prime}} \\
& \leq \frac{c^{\prime \prime}\|f\|_{2}^{\sigma}}{\left((1-r)^{-(1-p)-(1+\gamma)(1+p / 2) h(1-r))^{1 / q^{\prime}}}\right.}
\end{aligned}
$$

Now, we can apply Theorem 6.2 for the special function defined in (1.7).

Case 1. If $z_{n}=r_{n} e^{i \theta_{n}}, n \geq 1$, is a Carleson sequence satisfying

$$
\sum_{n=1}^{\infty}\left(1-r_{n}\right)^{\alpha}\left(\log \frac{1}{1-r_{n}}\right)^{\alpha_{1}} \cdots\left(\log _{m} \frac{1}{1-r_{n}}\right)^{\alpha_{m}}<\infty
$$

with $p \in(2 / 3,1), \sigma=2 p /(p+2),-1<\gamma<2 \sigma-2$, and $(1-p)+(1+\gamma)(1+p / 2)<$ $\alpha<p / 2$, then, for all $f \in K_{B}$, we have

$$
\begin{aligned}
& \left(\int_{0}^{1} \int_{0}^{2 \pi}\left|f^{\prime}\left(r \rho e^{i \theta}\right)\right|^{\sigma} \rho\left(1-\rho^{2}\right)^{\gamma} d \rho d \theta\right)^{1 / \sigma} \\
\leq & \frac{C\|f\|_{2}}{\left((1-r)^{\alpha-(1-p)-(1+\gamma)(1+p / 2)}\left(\log \frac{1}{1-r}\right)^{\alpha_{1}} \cdots\left(\log _{m} \frac{1}{1-r}\right)^{\alpha_{m}}\right)^{1 / p}}
\end{aligned}
$$

as $r \rightarrow 1^{-}$.

Case 2. If $z_{n}=r_{n} e^{i \theta_{n}}, n \geq 1$, is a Carleson sequence satisfying

$$
\sum_{n=1}^{\infty}\left(1-r_{n}\right)^{(1-p)+(1+\gamma)(1+p / 2)}\left(\log _{k} \frac{1}{1-r_{n}}\right)^{\alpha_{k}} \cdots\left(\log _{m} \frac{1}{1-r_{n}}\right)^{\alpha_{m}}<\infty,
$$

with $p \in(2 / 3,1), \sigma=2 p /(p+2),-1<\gamma<2 \sigma-2, \alpha_{k}, \alpha_{k+1}, \ldots, \alpha_{m} \in \mathbb{R}$, and $\alpha_{k}<$ 0 , then, for all $f \in K_{B}$, we have

$$
\left(\int_{0}^{1} \int_{0}^{2 \pi}\left|f^{\prime}\left(r \rho e^{i \theta}\right)\right|^{\sigma} \rho\left(1-\rho^{2}\right)^{\gamma} d \rho d \theta\right)^{1 / \sigma} \leq \frac{C\|f\|_{2}}{\left(\left(\log _{k} \frac{1}{1-r}\right)^{\alpha_{k}} \cdots\left(\log _{m} \frac{1}{1-r}\right)^{\alpha_{m}}\right)^{1 / p}}
$$

as $r \rightarrow 1^{-}$. However, if

$$
\sum_{n=1}^{\infty}\left(1-r_{n}\right)^{(1-p)+(1+\gamma)(1+p / 2)}<\infty
$$


then, we still have

$$
\left(\int_{0}^{1} \int_{0}^{2 \pi}\left|f^{\prime}\left(r \rho e^{i \theta}\right)\right|^{\sigma} \rho\left(1-\rho^{2}\right)^{\gamma} d \rho d \theta\right)^{1 / \sigma} \leq C\|f\|_{2},
$$

which means that

$$
f^{\prime} \in A_{\gamma}^{2 p /(p+2)}
$$

and the differential operator

$$
\begin{aligned}
K_{B} \longrightarrow A_{\gamma}^{2 p /(p+2)}, \\
f \longmapsto f^{\prime}
\end{aligned}
$$

is bounded.

Case 3. If $z_{n}=r_{n} e^{i \theta_{n}}, n \geq 1$, is a Carleson sequence satisfying

$$
\sum_{n=1}^{\infty}\left(1-r_{n}\right)^{p / 2}\left(\log _{k} \frac{1}{1-r_{n}}\right)^{\alpha_{k}} \cdots\left(\log _{m} \frac{1}{1-r_{n}}\right)^{\alpha_{m}}<\infty
$$

with $p \in(2 / 3,1), \sigma=2 p /(p+2),-1<\gamma<2 \sigma-2, \alpha_{k}, \alpha_{k+1}, \ldots, \alpha_{m} \in \mathbb{R}$ and $\alpha_{k}>0$, then, for all $f \in K_{B}$, we have

$$
\begin{aligned}
&\left(\int_{0}^{1} \int_{0}^{2 \pi}\left|f^{\prime}\left(r \rho e^{i \theta}\right)\right|^{\sigma} \rho\left(1-\rho^{2}\right)^{\gamma} d \rho d \theta\right)^{1 / \sigma} \\
&\left((1-r)^{(3 p / 2-1)-(1+\gamma)(1+p / 2)}\left(\log _{k} \frac{1}{1-r}\right)^{\alpha_{k}} \cdots\left(\log _{m} \frac{1}{1-r}\right)^{\alpha_{m}}\right)^{1 / p}
\end{aligned}
$$

as $r \rightarrow 1^{-}$. However, if

$$
\sum_{n=1}^{\infty}\left(1-r_{n}\right)^{p / 2}<\infty
$$

then, we still have

$$
\left(\int_{0}^{1} \int_{0}^{2 \pi}\left|f^{\prime}\left(r \rho e^{i \theta}\right)\right|^{\sigma} \rho\left(1-\rho^{2}\right)^{\gamma} d \rho d \theta\right)^{1 / \sigma} \leq \frac{C\|f\|_{2}}{(1-r)^{((3 p / 2-1)-(1+\gamma)(1+p / 2)) / p}}
$$

as $r \rightarrow 1^{-}$.

\section{REFERENCES}

1. P. R. Ahern and D. N. Clark, On inner functions with $H^{p}$-derivative, Michigan Math. J. 21 (1974), 115-127.

2. P. R. Ahern and D. N. Clark, On inner functions with $B^{p}$-derivative, Michigan Math. J. 23 (1976), 107-118.

3. W. S. Cohn, On the $H^{p}$ classes of derivative of functions orthogonal to invariant subspaces, Michigan Math. J. 30 (1983), 221-229.

4. P. L. Duren and A. Schuster, Bergman spaces, Math. Surveys and Monographs, 100 (Amer. Math. Soc., Providence, R.I., 2004).

5. P. L. Duren, Theory of $H^{p}$ spaces (Academic Press, 1970). 

$517-543$.

6. E. Fricain, Bases of reproducing kernels in model spaces, J. Operator Theory 46 (2001),

7. Y. Gotoh, On integral means of the derivatives of Blaschke products, Kodai Math. J. 30 (2007), 147-155.

8. v. Havin and J. Mashreghi, Admissible majorants for model subspaces of $H^{2}(\mathbb{R})$, Part I \& II, Canadian J. Math. 55, 6 (2003), 1231-1263 and 1264-1301.

9. H. Hedenmalm, B. Korenblum, and K. Zhu, Theory of Bergman spaces, Graduate Text in Mathematics, No. 199 (Springer-Verlag, 2000).

10. P. Koosis, Introduction to $H^{p}$ Spaces, Second Edition, Cambridge Tracts in Mathematics, No. 115 (Cambridge, 1998).

11. M. A. Kutbi, Integral means for the $n$ 'th derivative of Blaschke products, Kodai Math. J. 25 (2002), 191-208.

12. C. N. Linden, $H^{p}$-derivatives of Blaschke products, Michigan Math. J. 23 (1976), 43-51.

13. J. Mashreghi, Generalized Lipschitz functions, Comput. Methods Funct. Theory 5, (2005), 431-444.

14. N. Nikolski, Treatise on the shift operator (Springer-Verlag, 1986).

15. D. Protas, Blaschke products with derivatives in $H^{p}$ and $B^{p}$, Michigan Math. J. 20 (1973), 393-396.

16. D. Protas, Mean growth of the derivative of a Blaschke product, Kodai Math. J. 27 (2004), 354-359. 